\title{
Hypertriglyceridemia in Obese Children and Adolescents
}

\author{
Mo Kyung Jung, Eun-Gyong Yoo* \\ Department of Pediatrics, CHA Bundang Medical Center, CHA University, Seongnam, Korea
}

The increasing prevalence of obesity in children and adolescents is a serious public health concern. Hypertriglyceridemia is common in obese children and adolescents, and elevated triglyceride (TG) level is a known biomarker of cardiometabolic risk. Results from genetic studies suggest that TG and TG-rich lipoproteins and, more specifically, remnant cholesterol are in the causal pathway of cardiovascular disease. However, simultaneous measurement of all remnants has not yet been established, and plasma TG level can be used as a useful marker of remnant cholesterol. Adipose tissue dysfunction, including impaired adipocyte TG storage and release of fatty acids, mediates the development of obesity-related complications. The prevalence of hypertriglyceridemia increases in overweight or obese children and is associated with other cardiometabolic risk factors. Recently, the TG/high-density lipoprotein cholesterol (HDL-C) ratio was recognized as a marker of structural vascular changes and insulin resistance in obese youth. Recent guidelines recommend universal lipid screening with nonfasting non-HDL-C measurement in children at 9-11 years of age; however, fasting lipid profiles should be measured in obese children and overweight adolescents and in those with high non-HDL-C in universal screening. The primary approach to lower TG in children includes dietary and lifestyle modifications; however, children with severe hypertriglyceridemia should also be referred to a pediatric lipid specialist.

Received July 25, 2018

Reviewed August 9, 2018

Accepted September 12, 2018

${ }^{*}$ Corresponding author Eun-Gyong Yoo

(iD) https://orcid.org/0000-0002-6452-655X

Department of Pediatrics, CHA Bundang Medical Center, CHA University, 59 Yatap-ro, Bundang-gu, Seongnam 13496, Korea

Tel: +82-31-780-1959

Fax: +82-31-780-5239

E-mail: pedyoo@cha.ac.kr

Key words: Hypertriglyceridemia, Obesity, Child

\section{INTRODUCTION}

Obesity results from chronic energy imbalance involving dietary intake and physical activity. ${ }^{1}$ The increasing prevalence of obesity in children and adolescents is considered as one of the most series public health concerns in this century. ${ }^{2}$ Childhood obesity can adversely affect many organs and increases the risk of chronic diseases, including dyslipidemia, diabetes, and cardiovascular disease (CVD). ${ }^{2,3}$

According to a study that included 230,000 Norwegian adolescents, adolescent obesity was associated with increased mortality in middle age from several causes, including ischemic heart disease. ${ }^{4}$ Childhood body mass index (BMI) showed strong tracking to adulthood, and childhood BMI was independently related with ca-

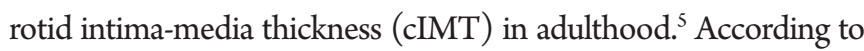
a large population-based U.S. study, the overall prevalence of dyslipidemia in normal weight adolescents was $14.2 \%$, which increased to $42.9 \%$ in obese youth. ${ }^{6}$ In obese adolescents, the prevalence of high triglyceride (TG), low high-density lipoprotein cholesterol (HDL-C), and high low-density lipoprotein cholesterol (LDL-C) was $24.1 \%, 20.5 \%$, and $14.2 \%$, respectively, suggesting that hypertriglyceridemia is one of the most common lipid abnormalities in obese youth. ${ }^{6} \mathrm{LDL}-\mathrm{C}$ is known to be a critical risk factor for early CVD and has been considered the primary target for risk reduction. ${ }^{7}$ However, patients treated with statins showed residual risk, and the most commonly observed lipid pattern in obese patients 
with CVD was high TG, low HDL-C, and normal to mildly elevated LDL-C. ${ }^{8}$

\section{TRIGLYCERIDES AND CARDIOVASCULAR RISK}

An elevated TG level is a known biomarker of cardiovascular risk, and hypertriglyceridemia is a component of metabolic syndrome. ${ }^{9,10}$ However, the specific role of TG has long been controversial, at least partially due to the high within-person variability compared to more stable factors such as HDL-C. ${ }^{10}$ Recent genetic studies suggest that TGs and TG-rich lipoproteins (TRLs) are causally related to CVD, rather than simply being a biomarker. ${ }^{11,12}$ Genome-wide association studies also reported causal associations between raised TGs and coronary artery disease. ${ }^{11,13}$ In comparison, a Mendelian randomization study showed that genetically reduced nonfasting plasma TG level leads to reduced mortality. ${ }^{14}$

Remnant cholesterol is defined as the cholesterol content of all TRLs. ${ }^{15}$ Simultaneous measurement of all remnants has not been established; however, remnant cholesterol can be calculated as HDL-C and LDL-C subtracted from nonfasting total cholesterol (TC). ${ }^{16}$ Even though plasma TG level is a useful marker of remnant cholesterol, it has been recently suggested that remnant cholesterol, rather than TGs, is in the causal pathway of atherosclerosis and CVD. ${ }^{16}$ Remnant lipoproteins can enter the arterial intima, similar to LDL, and remnants tend to be preferentially trapped in the intima, leading to inflammation, foam cell formation, atherosclerotic plaque formation, and finally CVD. ${ }^{16}$

\section{INSULIN RESISTANCE AND OBESITY-RELATED COMPLICATIONS}

Adipose tissue dysfunction, including impairment of adipocyte TG storage and release of fatty acids, mediates the development of obesity-related complications. ${ }^{17}$ Individuals with the ability to recruit healthy subcutaneous adipocytes in response to excess energy intake are relatively protected from obesity-associated morbidity. ${ }^{18}$ However, individuals with limited adipocyte recruitment ability will store excess fat in ectopic depots, such as the liver, visceral fat, and muscle, and are prone to metabolic complications of obesi- ty. $^{18,19}$

The difference in insulin sensitivity is related to different lipid partitioning patterns, and insulin resistance is associated with increased lipid deposition in the visceral and intramyocellular compartments. ${ }^{19,20}$ Intramyocellular and extramyocellular lipid deposition increased in obese adolescents and were directly associated with peripheral insulin sensitivity. ${ }^{21} \mathrm{~A}$ hypothesis has been suggested that unifies these concepts and proposes that the intracellular accumulation of lipids in muscle and liver triggers novel protein kinase $\mathrm{C}$ activation and subsequent insulin signaling impairment. ${ }^{21}$ This hypothesis accounts for the mechanism of insulin resistance in obesity and type 2 diabetes and the insulin-sensitizing effects of thiazolidinediones. 22

Visceral adiposity has also been reported to be related to atherogenic metabolic profiles in children. ${ }^{19}$ Intramyocellular lipid assessment in a clinical setting is not practical, but measuring waist circumference, an indirect measure of visceral fat, can easily be performed and used to identify children with higher metabolic risk. The hypertriglyceridemic waist phenotype, coexistence of elevated TG level and abdominal obesity, has been suggested to identify individuals at high risk for CVD. ${ }^{23}$

\section{HYPERTRIGLYCERIDEMIA IN CHILDREN AND ADOLESCENTS}

Studies on hypertriglyceridemia in children are currently limited. Recent reports on the prevalence of dyslipidemia in children and adolescents are shown in Table 1. In a large population-based U.S. study, the prevalence of hypertriglyceridemia was 5.9\% in normal weight children, which showed stepwise increase to $13.8 \%$ and $24.1 \%$ in overweight and obese children, respectively. ${ }^{6}$ The prevalence of hypertriglyceridemia in children and adolescents is reported to be $5.9 \%$ to $8.6 \%$ in the general population, which is comparable to other components of dyslipidemia. ${ }^{24-27}$ However, the prevalence of hypertriglyceridemia increases up to $13.8 \%-31.8 \%$ in overweight or obese children and adolescents and seems to be higher than other components of dyslipidemia, suggesting that hypertriglyceridemia could be the most common lipid abnormality among overweight or obese youth (Table 1).28-30

In a study of 139 Argentinian children, a status of overweight or 
Table 1. Summary of studies on the prevalence of dyslipidemia in children and adolescents

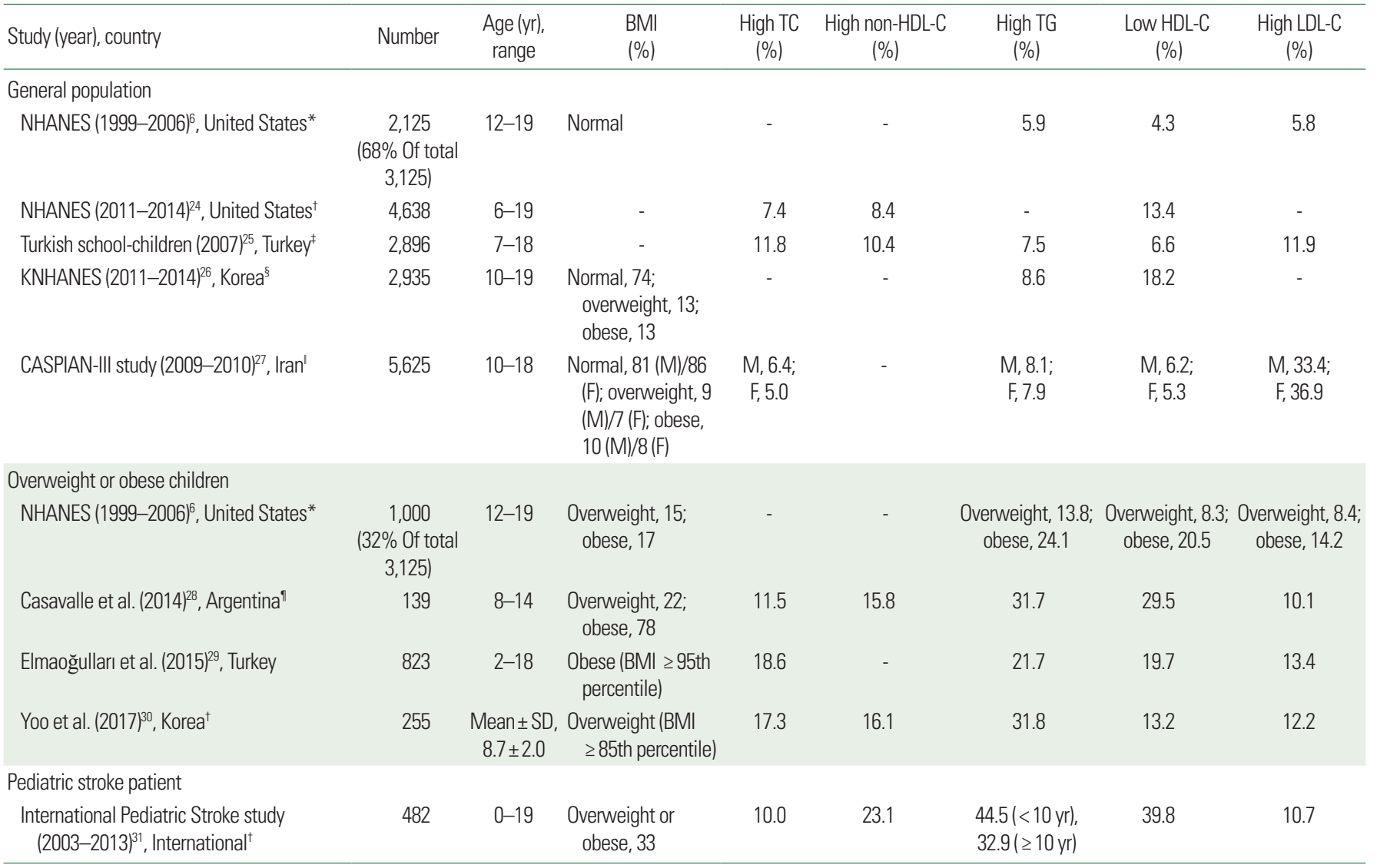

Cutoff values (mg/dL): *LDL-C $\geq 130$, low HDL-C $\leq 35$, and TG level $\geq 150$; ${ }^{\dagger} \mathrm{NHLBl}$ expert panel (2011): TC $\geq 200$, LDL-C $\geq 130$, non-HDL-C $\geq 145, H D L-C<40$, TG $\geq 100$ (0-9 years), and $\geq 130$ (10-19 years): ${ }^{\top} T C>200$, LDL-C $>130$, non-HDL-C >150, HDL-C $<35$, TG $>140$; ${ }^{5} T G \geq 150$, HDL-C $<40$ (boys aged $10-19$ years and girls $\leq 16$ years), and $<50$ (girls aged $\geq 16$ years); 'TC, LDL-C,TG, higher than the level corresponding to the age- and gender-specific 95th percentile, and/or HDL-C lower than the age- and gender-specific 5th percentile; ITC $\geq 200$, LDL-C $\geq 130$, non-HDL-C > 145, recommended by National Cholesterol Education Program.

BMI, body mass index; TC, total cholesterol; HDL-C, high-density lipoprotein cholesterol; TG, triglyceride; LDL-C, low-density lipoprotein cholesterol; NHANES, National Health and Nutrition Examination Survey; KNHANES, Korea National Health and Nutrition Examination Survey; CASPIAN, Childhood and Adolescence Surveillance and Prevention of Adult Noncommunicable Disease; M, male; F, female; SD, standard deviation.

obesity increased the odds ratios of hypertriglyceridemia, low HDL-C, and metabolic risk factors, and dyslipidemia was more commonly detected in subjects with central obesity, family history of dyslipidemia, and type 2 diabetes. ${ }^{28}$ Among 823 obese Turkish children and adolescents, the prevalence of dyslipidemia increased with increasing BMI, and hypertriglyceridemia was the most common type of dyslipidemia. ${ }^{29}$ Hepatosteatosis was more commonly observed in patients with dyslipidemia, and the level of insulin resistance was higher in subjects with both dyslipidemia and hepatosteatosis. $^{29}$

A study that included children in the International Pediatric Stroke study showed that dyslipidemia was more common in children with arterial ischemic stroke compared to the data from the
National Health and Nutrition Examination Survey (NHANES) (38.4\% vs. $21 \%$ ), and that $44.5 \%$ of children and $32.9 \%$ of adolescents with arterial ischemic stroke had hypertriglyceridemia, suggesting that the prevalence of dyslipidemia, including hypertriglyceridemia, might be higher in children with arterial ischemic stroke than in the general population. ${ }^{24,31}$

\section{TG/HDL-C RATIO AND RELATED FACTORS IN OBESE YOUTH}

Recent genetic studies suggested that low HDL-C is not causally related to CVD, but is likely an innocent bystander. ${ }^{13,15}$ However, most studies in children and adolescents report the associations 
Table 2. Summary of recent studies on TG/HDL-C ratio in children and adolescents

\begin{tabular}{|c|c|c|}
\hline Author (year), country & Number (age, yr*) & Result \\
\hline Giannini et al. $(2011)^{32}$, United States & $1,452(13.1 \pm 2.9)$ & $\begin{array}{l}\text { The TG/HDL-C ratio was associated with insulin resistance in obese Caucasian youth, and the OR for insu- } \\
\text { lin resistance was } 6.02 \text { in those with TG/HDL-C higher than } 2.27 \text {. }\end{array}$ \\
\hline Yoo et al. $(2017)^{30}$, Korea & $\begin{array}{l}769 \text { (overweight, } 8.7 \pm 2.0 \text {; } \\
\text { normal, } 8.9 \pm 1.8 \text { ) }\end{array}$ & $\begin{array}{l}\text { The TG/HDL-C ratio correlated with HOMA-IR in overweight children ( } r=0.282, P=0.016) \text {. The specificity of } \\
\text { high TG/HDL-C was better than that of hypertriglyceridemia for identifying overweight children with high } \\
\text { HOMA-IR ( } 72.9 \% \text { vs. } 64.2 \%) \text {. }\end{array}$ \\
\hline KNHANES $(2011-2014)^{26}$, Korea & $2,935(10-19)$ & TG/HDL-C ratio (0.891 [95\% Cl, 0.891-0.892]) showed the highest predictability for CMRF clustering. \\
\hline Pacifico et al. (2014) ${ }^{33}$, Italy & $548(6-16)$ & $\begin{array}{l}\text { Increased cIMT was associated with high TG/HDL-C ratio }(0 \mathrm{R}, 1.81 ; 95 \% \mathrm{Cl}, 1.08-3.04 ; P<0.05) \text { in a step- } \\
\text { wise multivariate logistic regression analysis. }\end{array}$ \\
\hline Urbina et al. $(2013)^{34}$, United States & $893(10-26)$ & The high TG/HDL-C ratio group had the stiffest vessels (all $P<0.03$, based on analysis of variance). \\
\hline CARITALY study (2003-2013) $)^{35}$, Italy & $5,505(5-18)$ & $\begin{array}{l}\text { The ORs for insulin resistance, high blood pressure, metabolic syndrome, presence of liver steatosis, and } \\
\text { increased cIMT were higher in children with high TG/HDL-C compared to children with high non-HDL-C. }\end{array}$ \\
\hline
\end{tabular}

*Values are presented as mean \pm standard deviation or range.

TG/HDL-C, triglyceride to high-density lipoprotein cholesterol; HOMA-IR, homeostasis model assessment of insulin resistance; KNHANES, Korea National Health and Nutrition Examination Survey; Cl, confidence interval; CMRF, cardiometabolic risk factor; MetS, metabolic syndrome; cIMT, carotid intima-media thickness; OR, odds ratio; CARITALY, CARdiometabolic risk factors in overweight and obese children in ITALY.

between TG/HDL-C ratio, not TG itself, and metabolic risk factors. Table 2 summarizes recent reports on the TG/HDL-C ratio and related factors in children and adolescents.

The TG/HDL-C ratio was associated with insulin resistance in obese Caucasian youth, and the odds ratio for insulin resistance was 6.02 in those with high TG/HDL-C $(>2.27) .{ }^{32}$ Our previous study also showed that TG/HDL-C ratio $(\geq 2.0)$ showed higher sensitivity (55.6\%) and specificity (72.9\%) than TC, non-HDL-C, and LDL-C in identifying overweight children with increased homeostasis model assessment of insulin resistance (HOMA-IR) ${ }^{30} \mathrm{~A}$ recent study that used data for 2,935 adolescents from the 2011 to 2014 Korean NHANES reported that TG/HDL-C ratio showed the highest predictability for clustering of cardiometabolic risk factors (Table 2). ${ }^{26}$

The TG/HDL-C ratio was recognized as a suggestive marker of structural vascular changes and insulin resistance in obese youth. A study on the association of TG/HDL ratio with cIMT in children and adolescents showed that the odds ratios for insulin resistance increased with increasing TG/HDL-C ratio tertile, and that increased cIMT was independently associated with a high TG/ HDL-C ratio. ${ }^{33}$ A longitudinal school-based study indicated that the high TG/HDL-C ratio was an independent determinant of arterial stiffness in obese youth. ${ }^{34}$ Additionally, a recent study suggested that a TG/HDL-C ratio $\geq 2.2$ can be used as an accurate screening parameter of insulin resistance, metabolic syndrome, and increased cIMT (Table 2). ${ }^{35}$

\section{LIPID SCREENING AND DYSLIPIDEMIA DIAGNOSIS IN CHILDREN AND ADOLESCENTS}

Non-HDL-C, calculated as TC minus HDL-C, reflects the sum of highly atherogenic lipoprotein particles. ${ }^{36}$ Recent guidelines recommend universal lipid screening with nonfasting non-HDL-C measurement in all youth between the ages of 9-11 and 17-21 years. ${ }^{37}$ If the non-HDL-C is $145 \mathrm{mg} / \mathrm{dL}$ or higher, a fasting lipid profile measurement should be performed. ${ }^{37}$ Lipid screening of dyslipidemia is not recommended for ages less than 2 years. However, a fasting lipid profile should be measured in obese children (BMI $\geq 95$ th percentile) aged 2-8 years and in overweight adolescents (BMI $\geq 85$ th percentile) aged 12-16 years, and those in these age group with a family history of myocardial infarction, stroke before the age of 55 in males or the age of 65 in females, or risk factors such as hypertension, diabetes mellitus, Kawasaki disease, or nephrotic syndrome. ${ }^{38}$

Hypertriglyceridemia can be diagnosed if TG level is $\geq 100 \mathrm{mg} / \mathrm{dL}$ in children ( $<10$ year) or $\geq 130 \mathrm{mg} / \mathrm{dL}$ in adolescents (10-19 year) based on an average of two fasting measurements. ${ }^{37}$ Ethnic differences in TG level and related factors have been reported, and further investigation is needed to develop ethnicity-specific cutoffs. ${ }^{32}$ 
Secondary causes of hypertriglyceridemia, such as hypothyroidism, polycystic ovarian syndrome, and medication effects, should also be ruled out. ${ }^{36} \mathrm{~A}$ correction between underlying causes could result in normalization of TG level for all of these cases.

\section{PEDIATRIC HYPERTRIGLYCERIDEMIA MANAGEMENT}

The primary approach for reducing and managing TG in children includes dietary and lifestyle modifications. ${ }^{36}$ In addition, the primary aim of hypertriglyceridemia management is weight control for obese children and adolescents. Reduced caloric intake with or without increased exercise resulted in significant reduction of TG level as well as weight loss and improvement of other metabolic profiles..$^{39}$ In another report from the Young Finns study, childhood dietary patterns remained stable over the life course and were associated with cardiovascular risk factors and measures of subclinical atherosclerosis, suggesting that childhood nutrition might have significant influence on the progression of CVD. ${ }^{40}$

Dietary modification with a cardiovascular health integrated lifestyle diet (CHILD)-1 and then CHILD-2 TG should be recommended for children with hypertriglyceridemia. ${ }^{33,37}$ Simple sugars need to be substituted for complex carbohydrates, sugar-sweetened beverages should be excluded, and fish consumption is encouraged to increase the intake of omega- 3 fatty acids. ${ }^{36}$ Lifestyle changes, including increasing physical activity to 60 minutes per day, reducing screen time to less than 2 hours per day, attaining ideal body weight ( $\leq 85$ th percentile), and optimizing blood pressure, should be encouraged for all patients with increased TG level. ${ }^{36,37}$

Medication is rarely indicated for children with hypertriglyceridemia who respond well to weight loss and lifestyle changes. ${ }^{37} \mathrm{How}$ ever, children with severe hypertriglyceridemia (TG $>500 \mathrm{mg} / \mathrm{dL}$ ) should be referred to a pediatric lipid specialist, and the symptoms of pancreatitis should be counseled. ${ }^{41}$ Although fibric acid derivatives are commonly used as TG lowering agents in adults, these have not yet been approved for children by the Food and Drug Administration (FDA). ${ }^{37,42}$ Although a high dose of omega-3 fatty acids has been shown to decrease TG level by $20 \%-30 \%$ in adults, prescription of omega- 3 fatty acids for children is not currently approved by the U.S. FDA. ${ }^{43}$

\section{CONCLUSION}

Hypertriglyceridemia is common in obese children and adolescents and may increase the patient's future cardiovascular risk. Although universal screening with nonfasting non-HDL cholesterol is recommended, TGs should also be measured in obese children and adolescents. Early detection and intervention with lifestyle modification have been helpful approaches for obese children and adolescents with increased TG level.

\section{CONFLICTS OF INTEREST}

The authors declare no conflict of interest.

\section{REFERENCES}

1. Gortmaker SL, Swinburn BA, Levy D, Carter R, Mabry PL, Finegood DT, et al. Changing the future of obesity: science, policy, and action. Lancet 2011;378:838-47.

2. Güngör NK. Overweight and obesity in children and adolescents. J Clin Res Pediatr Endocrinol 2014;6:129-43.

3. Han JC, Lawlor DA, Kimm SY. Childhood obesity. Lancet 2010;375:1737-48.

4. Bjørge T, Engeland A, Tverdal A, Smith GD. Body mass index in adolescence in relation to cause-specific mortality: a followup of 230,000 Norwegian adolescents. Am J Epidemiol 2008; 168:30-7.

5. Freedman DS, Patel DA, Srinivasan SR, Chen W, Tang R, Bond $\mathrm{MG}$, et al. The contribution of childhood obesity to adult carotid intima-media thickness: the Bogalusa Heart Study. Int J Obes (Lond) 2008;32:749-56.

6. Centers for Disease Control and Prevention (CDC). Prevalence of abnormal lipid levels among youths: United States, 1999-2006. MMWR Morb Mortal Wkly Rep 2010;59:29-33.

7. Expert Panel on Detection, Evaluation, and Treatment of High Blood Cholesterol in Adults. Executive summary of the third report of the National Cholesterol Education Program (NCEP) expert panel on detection, evaluation, and treatment of high blood cholesterol in adults (Adult Treatment Panel III). JAMA 2001;285:2486-97. 
8. Kavey RE, Mietus-Snyder M. Beyond cholesterol: the atherogenic consequences of combined dyslipidemia. J Pediatr 2012; 161:977-9.

9. Alberti KG, Zimmet P, Shaw J. Metabolic syndrome: a new world-wide definition. A consensus statement from the international diabetes federation. Diabet Med 2006;23:469-80.

10. Boullart AC, de Graaf J, Stalenhoef AF. Serum triglycerides and risk of cardiovascular disease. Biochim Biophys Acta 2012; 1821:867-75.

11. Budoff M. Triglycerides and triglyceride-rich lipoproteins in the causal pathway of cardiovascular disease. Am J Cardiol 2016;118:138-45.

12. Lewis GF, Xiao C, Hegele RA. Hypertriglyceridemia in the genomic era: a new paradigm. Endocr Rev 2015;36:131-47.

13. Do R, Willer CJ, Schmidt EM, Sengupta S, Gao C, Peloso GM, et al. Common variants associated with plasma triglycerides and risk for coronary artery disease. Nat Genet 2013;45:1345-52.

14. Thomsen M, Varbo A, Tybjærg-Hansen A, Nordestgaard BG. Low nonfasting triglycerides and reduced all-cause mortality: a mendelian randomization study. Clin Chem 2014;60:737-46.

15. Varbo A, Benn M, Tybjærg-Hansen A, Jørgensen AB, FrikkeSchmidt R, Nordestgaard BG. Remnant cholesterol as a causal risk factor for ischemic heart disease. J Am Coll Cardiol 2013; 61:427-36.

16. Nordestgaard BG, Varbo A. Triglycerides and cardiovascular disease. Lancet 2014;384:626-35.

17. Halberg N, Wernstedt-Asterholm I, Scherer PE. The adipocyte as an endocrine cell. Endocrinol Metab Clin North Am 2008; 37:753-68.

18. Bray GA, Heisel WE, Afshin A, Jensen MD, Dietz WH, Long $M$, et al. The science of obesity management: an endocrine society scientific statement. Endocr Rev 2018;39:79-132.

19. Weiss R, Kaufman FR. Metabolic complications of childhood obesity: identifying and mitigating the risk. Diabetes Care 2008; 31 Suppl 2:S310-6.

20. Weiss R, Dufour S, Taksali SE, Tamborlane WV, Petersen KF, Bonadonna RC, et al. Prediabetes in obese youth: a syndrome of impaired glucose tolerance, severe insulin resistance, and altered myocellular and abdominal fat partitioning. Lancet 2003; 362:951-7.
21. Sinha R, Dufour S, Petersen KF, LeBon V, Enoksson S, Ma YZ, et al. Assessment of skeletal muscle triglyceride content by (1) $\mathrm{H}$ nuclear magnetic resonance spectroscopy in lean and obese adolescents: relationships to insulin sensitivity, total body fat, and central adiposity. Diabetes 2002;51:1022-7.

22. Samuel VT, Petersen KF, Shulman GI. Lipid-induced insulin resistance: unravelling the mechanism. Lancet 2010;375:226777.

23. Lemieux I, Pascot A, Couillard C, Lamarche B, Tchernof A, Alméras N, et al. Hypertriglyceridemic waist: a marker of the atherogenic metabolic triad (hyperinsulinemia; hyperapolipoprotein B; small, dense LDL) in men? Circulation 2000;102: 179-84.

24. Nguyen D, Kit B, Carroll M. Abnormal cholesterol among children and adolescents in the United States, 2011-2014. NCHS Data Brief 2015;(228):1-8.

25. Uçar B, Kiliç Z, Dinleyici EC, Colak O, Güneş E. Serum lipid profiles including non-high density lipoprotein cholesterol levels in Turkish school-children. Anadolu Kardiyol Derg 2007;7: 415-20.

26. Seo JY, Kim JH. Validation of surrogate markers for metabolic syndrome and cardiometabolic risk factor clustering in children and adolescents: a nationwide population-based study. PLoS One 2017;12:e0186050.

27. Kelishadi R, Jamshidi F, Qorbani M, Motlagh ME, Heshmat R, Ardalan G, et al. Association of hypertriglyceridemic-waist phenotype with liver enzymes and cardiometabolic risk factors in adolescents: the CASPIAN-III study. J Pediatr (Rio J) 2016;92:512-20.

28. Casavalle PL, Lifshitz F, Romano LS, Pandolfo M, Caamaño A, Boyer PM, et al. Prevalence of dyslipidemia and metabolic syndrome risk factor in overweight and obese children. Pediatr Endocrinol Rev 2014;12:213-23.

29. Elmaoğulları S, Tepe D, Uçaktürk SA, Karaca Kara F, Demirel F. Prevalence of dyslipidemia and associated factors in obese children and adolescents. J Clin Res Pediatr Endocrinol 2015; 7:228-34.

30. Yoo DY, Kang YS, Kwon EB, Yoo EG. The triglyceride-to-high density lipoprotein cholesterol ratio in overweight Korean children and adolescents. Ann Pediatr Endocrinol Metab 2017; 


\section{2:158-63.}

31. Sultan S, Dowling M, Kirton A, DeVeber G, Linds A, Elkind MS, et al. Dyslipidemia in children with arterial ischemic stroke: prevalence and risk factors. Pediatr Neurol 2018;78:46-54.

32. Giannini C, Santoro N, Caprio S, Kim G, Lartaud D, Shaw M, et al. The triglyceride-to-HDL cholesterol ratio: association with insulin resistance in obese youths of different ethnic backgrounds. Diabetes Care 2011;34:1869-74.

33. Pacifico L, Bonci E, Andreoli G, Romaggioli S, Di Miscio R, Lombardo CV, et al. Association of serum triglyceride-to-HDL cholesterol ratio with carotid artery intima-media thickness, insulin resistance and nonalcoholic fatty liver disease in children and adolescents. Nutr Metab Cardiovasc Dis 2014;24: 737-43.

34. Urbina EM, Khoury PR, McCoy CE, Dolan LM, Daniels SR, Kimball TR. Triglyceride to HDL-C ratio and increased arterial stiffness in children, adolescents, and young adults. Pediatrics 2013;131:e1082-90.

35. Di Bonito P, Valerio G, Grugni G, Licenziati MR, Maffeis C, Manco M, et al. Comparison of non-HDL-cholesterol versus triglycerides-to-HDL-cholesterol ratio in relation to cardiometabolic risk factors and preclinical organ damage in overweight/obese children: the CARITALY study. Nutr Metab Cardiovasc Dis 2015;25:489-94.

36. Bamba V. Update on screening, etiology, and treatment of dyslipidemia in children. J Clin Endocrinol Metab 2014;99: 3093-102.

37. Expert Panel on Integrated Guidelines for Cardiovascular Health and Risk Reduction in Children and Adolescents; National Heart, Lung, and Blood Institute. Expert panel on integrated guidelines for cardiovascular health and risk reduction in children and adolescents: summary report. Pediatrics 2011; 128 Suppl 5:S213-56.

38. Yang S, Hwang JS, Park HK, Lee HS, Kim HS, Kim EY, et al. Serum lipid concentrations, prevalence of dyslipidemia, and percentage eligible for pharmacological treatment of Korean children and adolescents: data from the Korea National Health and Nutrition Examination Survey IV (2007-2009). PLoS One 2012;7:e49253.

39. Ho M, Garnett SP, Baur LA, Burrows T, Stewart L, Neve M, et al. Impact of dietary and exercise interventions on weight change and metabolic outcomes in obese children and adolescents: a systematic review and meta-analysis of randomized trials. JAMA Pediatr 2013;167:759-68.

40. Kaikkonen JE, Mikkilä V, Magnussen CG, Juonala M, Viikari JS, Raitakari OT. Does childhood nutrition influence adult cardiovascular disease risk? Insights from the Young Finns Study. Ann Med 2013;45:120-8.

41. Murad MH, Hazem A, Coto-Yglesias F, Dzyubak S, Gupta S, Bancos I, et al. The association of hypertriglyceridemia with cardiovascular events and pancreatitis: a systematic review and meta-analysis. BMC Endocr Disord 2012;12:2.

42. Berglund L, Brunzell JD, Goldberg AC, Goldberg IJ, Sacks F, Murad MH, et al. Evaluation and treatment of hypertriglyceridemia: an Endocrine Society clinical practice guideline. J Clin Endocrinol Metab 2012;97:2969-89.

43. Skulas-Ray AC, Kris-Etherton PM, Harris WS, Vanden Heuvel JP, Wagner PR, West SG. Dose-response effects of omega-3 fatty acids on triglycerides, inflammation, and endothelial function in healthy persons with moderate hypertriglyceridemia. Am J Clin Nutr 2011;93:243-52. 